\title{
Parkinson's disease permanent care unit: managing the chronic-palliative interface
}

This article was published in the following Dove Press journal:

Journal of Multidisciplinary Healthcare

3I March 20II

Number of times this article has been viewed

\section{Johan Lökk}

Department of Neurobiology, Caring Sciences, and Society, Karolinska Institutet; Geriatric Department, Karolinska University Hospital Huddinge, Stockholm, Sweden
Correspondence: Johan Lökk Geriatric Dept, Karolinska University Hospital Huddinge, Stockholm, Sweden Tel +4685858000

Fax +46858585482

Email johan.lokk@karolinska.se
Background: Parkinson's disease (PD) eventually leads to severe functional decline and dependence. Specialized care units for PD patients in need of permanent care are lacking.

Methods: Patients with severe PD are referred to the PD permanent care unit harboring 30 patients with specialized medical and health care provided by trained staff. Patients need to have intensive medical and care needs, and be no longer able to stay at home or at an ordinary institution. A written and continuously reviewed care plan is made for each patient at admission, with the overriding aim to preserve quality of life and optimize functionality.

Results: After five years, the PD permanent care unit has cared for 70 patients (36 men and 34 women) with a mean age of 76.6 years and a mean duration of Parkinsonism of 11.8 years. Hoehn and Yahr severity of disease was 3.7, cognition was 25.3 (Mini-Mental State Examination), and the mean daily levodopa dose was $739 \mathrm{mg}$. The yearly fatality rate was seven, and the mean duration of stay was 26.9 months. Only five patients moved out from the unit.

Conclusion: A specially designed and staffed care unit for Parkinsonism patients seems to fill a need for patients and caregivers, as well as for social and health care authorities. This model is sensitive to the changing needs and capacities of patients, ensuring that appropriate services are available in a timely manner. There was a rather short duration of patient stay and remaining life span after admission to the unit. Despite the chronic/palliative state of patients at the PD permanent care unit, there are many therapeutic options, with the overriding objective being to allow the patients to end their days in a professional and comfortable environment.

Keywords: Parkinsonism, palliative care, end-stage disease, permanent care

\section{Introduction}

The most common neurodegenerative movement disorder is Parkinson's disease (PD), eventually leading to severe functional decline and dependence. ${ }^{1}$ During disease progression, patients develop not only motor complications, like fluctuations and dyskinesia, but also nonmotor symptoms from many organs., ${ }^{2,3}$ These nonmotor symptoms are those that do not traditionally count as PD symptoms, but are receiving more interest in recent years because PD today is recognized as a multisystem brain disorder. The longer the duration of disease, the more the accumulation of these symptoms, which are often more devastating to patients than the motor symptoms. Neuropsychiatric symptoms like nightmares, paranoid ideas, hallucinations, and, ultimately, psychosis, often accompanied by cognitive decline, may appear. ${ }^{4}$ These symptoms are often very troublesome to the patient as well as to the caregiver, and are sometimes so difficult to treat and cope with, that affected patients are eventually institutionalized. ${ }^{5}$ This occurs even if the patients have devoted caregivers, as well as formal home help services. 
From disease onset and/or diagnosis, most patients are treated and cared for at outpatient departments. However, with PD being a progressive disorder, the worsening of and onset of new symptoms increases the need for outpatient visits, temporary hospital stays, home help, and respite care. It is evident that PD patients, unlike many other vulnerable patient groups, eg, those with Alzheimer's disease or stroke, are often excluded from permanent stay in special care units. Such a unit could be an appropriate site when the disease is at an end-stage and palliative phase, the caregivers are no longer able to care for the patient, and more home help is not possible or adequate. This should be appropriate because the view of palliative care is now broader, and includes care for those patients in whom the consequences of their illness require treatment, regardless of the prognosis of the patient. ${ }^{6}$ Thus, palliation is not the same as end-of-life care, care targeted for cancer patients, or patients with a bad prognosis. When it comes to complex neurodegenerative diseases like $\mathrm{PD}$, it is difficult to use prognosis estimates as determining factors for referrals to palliative care, ${ }^{7}$ and even if there is a need for palliative care for a PD patient, such care is poorly developed. ${ }^{8}$

Therefore, there was a need for patients, caregivers, patient organizations, and social authorities to organize a special care unit for PD patients in need of permanent care with specially trained and interested staff. Such a specialized PD care unit for permanent stay was launched in 2004 and, to our knowledge, no such unit was then currently existing in Europe. The organization and launching of the unit was preceded by negotiations and agreements between local health and social care authorities, a housing company, and the Swedish Parkinson Disease Organization leading to joint liability for lodging, caring, and medical health services. We report here the general design, implementation, and results since starting five years ago.

\section{Methods}

The Parkinsonism permanent care unit is situated in the center of Täby, a suburb of Stockholm. Patients with a diagnosis of Parkinsonism living within the Stockholm catchment area of about 2,020,000 inhabitants are eligible to be referred to the unit. Referred patients are carefully assessed by two specially trained nurses, as well as representatives from the Stockholm social care authorities, in order to assess the patient's needs and grant a permit for permanent care for the patient before admission. Referrals are issued by physicians or representatives of social care authorities with the prerequisite that the patient has intense medical and caring needs and, due to these, is no longer able to stay at home or at an ordinary nursing home. Patients with severe psychotic or behavioral symptoms making it impossible to stay within the unit and those at risk of hurting others are the only ones who are ineligible.

The PD permanent care unit houses 30 patients, all in their own rooms with options to do their own furnishing. There are shared dining and living rooms, as well as training facilities within the unit. Patients have their meals together at regular times, with the option for patients to assist the staff in meal preparation in order to maintain normal activities of daily living as far as possible.

The staff comprises two nurses and ten assistant nurses during the day time, six assistant nurses in the evenings until $10 \mathrm{pm}$, and two at night time. A physician, ie, a movement disorder specialist, visits the PD permanent care unit every fourth week and then, according to a preset schedule, assesses ten patients each time, as well as on request. There is also a geriatrician who visits the PD permanent care unit weekly, taking care of general medical problems. Moreover, an occupational therapist and a physiotherapist work parttime on a daily basis at the unit. Before the opening of the PD permanent care unit, the staff had been carefully selected and then educated for six weeks regarding symptomatology, treatment, and care of PD.

A written and continuously reviewed care plan with a multidisciplinary approach is made for each patient at admission. This includes recognition of the patient's medical and care status, as well as their social, emotional, cultural, and spiritual needs. It also means determining and regularly assessing these, and whether the PD permanent care unit is the appropriate site of care. Severity of disease is measured by Hoehn and Yahr ${ }^{9}$ and cognition by Mini-Mental State Examination. ${ }^{10}$ Hoehn and Yahr is an ordinal scale categorizing the severity of PD from 0 to 5 , where 0 represents mild disease and 5 very severe disease. All services aim to preserve the patient's quality of life and optimize functionality as much as possible, as well as to alleviate problematic symptoms whatever their cause, with a supporting and comforting attitude from caring staff. Physical, mental, and emotional changes in the patient are handled at the PD permanent care unit as much as possible, with the possibility of a temporary transfer to hospital if there are other illnesses demanding specialized investigations or treatments. However, patients are to be readmitted to the PD permanent care unit after the acute phase is over.

Common nonmotor symptoms are handled individually, guided by a special treatment protocol (Table 1$).{ }^{11}$ In addition 
Table I Treatment guide for some nonmotor symptoms in Parkinson's disease

\begin{tabular}{ll}
\hline Insomnia & Adjust anti-PD drugs, sleep hygiene, zolpidem, clonazepam \\
Depression & Serotonin and noradrenergic reuptake inhibitors, amitryptiline, mirtazapine \\
Rapid eye movement behavior disorder & Adjust anti-PD drugs, clonazepam \\
Fatigue & Amantidine, modafinil \\
Day time sleepiness & Modafinil, selegiline \\
Psychosis, hallucinations & Adjust anti-PD drugs, rivastigmine (mild); atypical antipsychotic drugs, clozapine, quetiapine, \\
& lanzapine (severe) \\
Cognitive impairment, dementia & Rivastigmine \\
Constipation & Osmotic laxatives, macrogol \\
Urinary urgency & Check all drugs, anticholinergic bladder stabilizers, desmopressin for nocturia \\
Impotence & Sildenafil, tadafil \\
Pain & Adjust anti-PD drugs, muscle relaxants, paracetamol \\
Restless legs & Dopamine agonists \\
Orthostatic hypotension & Adjust anti-PD drugs, increase water and salt intake, fludrocortisone, ephedrine, midodrine \\
Drooling & $0.5 \%$ atropine eye drops sublingually, scopoderm patch, botulinum toxin injections into \\
& salivary glands \\
Excessive sweating & Adjust anti-PD drugs, propantheline, propranolol, topical aluminum creams \\
\hline
\end{tabular}

Abbreviation: PD, Parkinson's disease.

to the risk of developing or having cognitive failure, another common and pervasive complication in late-stage PD is postural instability. This may create a fear of falling, resulting in dependency and immobilization, which might increase the risk of constipation and osteoporosis. ${ }^{11}$ Physiotherapy and exercise to improve gait, balance, joint mobility, and transfers are performed to compensate for debilitating disease progression. ${ }^{12}$

Patients are thoroughly assessed at admission to the PD permanent care unit, and screened with laboratory tests on a yearly basis thereafter, as well as continuously followed up regarding their Parkinsonism, especially their nonmotor symptoms. Weight, appetite, stool, and risk of falls, ${ }^{13}$ as well as neuropsychiatric problems and sleep, are followed up on a daily basis, with adequate action plans when needed. Family members are also incorporated in the patient's care planning and regular family meetings are established, thus informing and involving family members and assessing their needs and wishes. The study was approved by the regional ethics committee of Stockholm.

\section{Results}

Over a period of five years, the PD permanent care unit has cared for 70 patients (36 men and 34 women) of mean age 76.6 (range 60-90) years at admission. The mean duration of Parkinsonism was 11.8 (range 3-29) years, with a mean Hoehn and Yahr of 3.7 (range 1-5) and a mean Mini-Mental State Examination of 23.8 (range 10-30, see Table 2). Some activities of daily living-dependent factors as surrogate measures of caregiver burden are also depicted in Table 2 .

There were 62 patients with a diagnosis of idiopathic PD, three with multiple system atrophy, two with progressive supranuclear palsy, one with corticobasal degeneration, one with dementia with Lewy bodies, and one with vascularinduced Parkinsonism. The initial PD diagnosis was later on reassessed and rediagnosed as multiple system atrophy in two cases and dementia with Lewy bodies in one case. The mean daily levodopa dose was 739 (range 300-1200) mg, with 16 patients having additional therapy with a catechol-O-methyl transferase inhibitor, 14 patients with a monoamine oxidase $\mathrm{B}$ inhibitor, 12 patients with a dopamine agonist, one patient with Duodopa $\AA$, and three with deep brain stimulation. The mean duration of stay was 26.9 (range 1-58) months, where five patients moved out from the PD permanent care unit for social reasons after a mean length of stay of six (range 1-13) months. There were 13 acute transfers to hospital due to exacerbation or new onset of comorbidity, but none due to Parkinsonism per se. The mean annual fatality rate was seven (range 5-8) with pneumonia as the most common cause of death. There were no significant gender differences for the parameters described (Table 2). During the last three years, there has been a waiting list for admission of 2.5 months.

\section{Discussion}

A specially designed and staffed care unit, exclusively for patients with Parkinsonism, appears to fill a need for both patients and caregivers, as well as for social and health care authorities. Patients are in a complicated chronic/palliative state of their disease, which is a prerequisite for being referred and accepted to the PD permanent care unit. They are mostly elderly patients, with a PD duration of more than ten years, and a rather high caregiver burden, as reflected by high Hoehn and Yahr scores, a high number of neuropsychiatric symptoms and medications, and a need for help in activities 
Table 2 Characteristics of patients at the Parkinson's disease permanent care unit 2005-2009

\begin{tabular}{|c|c|c|c|c|c|}
\hline & \multirow{2}{*}{$\frac{\text { All }(n=70)}{\text { Mean }}$} & \multicolumn{2}{|c|}{ Males $(n=36)$} & \multicolumn{2}{|c|}{ Females $(n=34)$} \\
\hline & & Mean & Range & Mean & Range \\
\hline Age (years) & 76.6 & 76.0 & $60-86$ & 77.2 & $64-90$ \\
\hline Duration of PD (years) & 11.79 & 12.19 & $3-28$ & 11.35 & $3-35$ \\
\hline Hoehn and Yahr & 3.7 & 3.6 & $2-5$ & 3.8 & $\mathrm{I}-5$ \\
\hline Levodopa (mg) & 739 & 735 & $300-1100$ & 743 & $300-1200$ \\
\hline Duration at PPCU (years) & 26.9 & 25.4 & $3-53$ & 28.6 & $\mathrm{I}-58$ \\
\hline MMT & 23.8 & 24.1 & $10-30$ & 23.7 & $10-30$ \\
\hline Falls/year & 63 & 35 & $|9-5|$ & 28 & $18-40$ \\
\hline Incontinence & 57 & 35 & & 22 & \\
\hline Wheelchair & 50 & 27 & & 23 & \\
\hline Feeding help & 28 & 15 & & 13 & \\
\hline No ADL help & 7 & 3 & & 4 & \\
\hline Total ADL help & 25 & 15 & & 10 & \\
\hline Antidepressant use & 28 & 10 & & 18 & \\
\hline Antipsychotic use & 23 & 9 & & 14 & \\
\hline
\end{tabular}

Abbreviations: ADL, activities of daily living; MMT, methylcyclopentadienyl manganese tricarbonyl; PD, Parkinson's disease; PPCU, Parkinson's disease permanent care unit.

of daily living, as indicated by the need for a wheelchair and help with feeding (see Figure 1).

Parkinsonism was the inclusion criterion, and not only that arising from idiopathic PD, because the need for and approach to treatment would be the same for all affected patients. However, the majority of our patients had a diagnosis of idiopathic PD. Only a few patients have been permanently discharged from the unit during the first five years of the unit's operation, and those who actually moved out from the PD permanent care unit did so for social and not medical reasons, mostly in order to be closer to their relatives. However, the vast majority of patients remained at the unit until the end of their lives. Surprisingly, there was a rather short duration of stay and remaining life span for these patients after admission to the unit, despite the fact that they had had Parkinsonism for about ten years, but were now in a severe stage of disease. Not unexpectedly, the patients deteriorated during their stay, having more severe symptoms as well as concomitant diseases. Other studies have highlighted the complications experienced by PD patients and pointed out

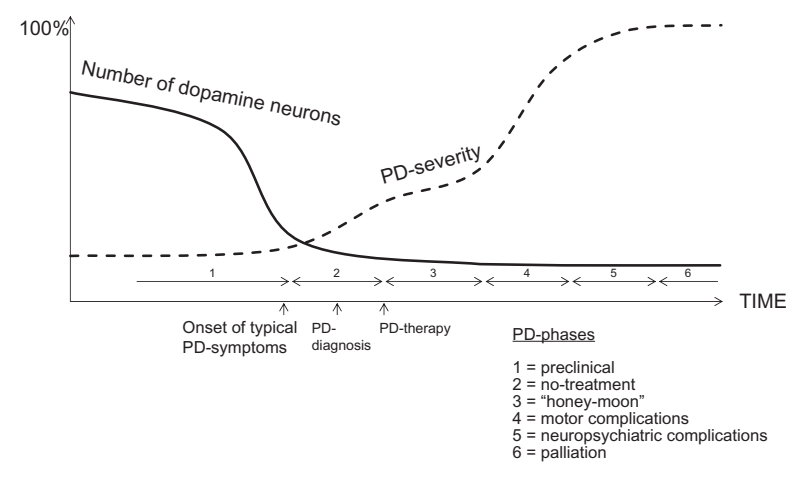

Figure I Schematic view of PD progression and its different stages. strategies to maintain symptom control in late-stage PD. ${ }^{14,15}$ Even cognitive behavior therapy has been tried in PD patients suffering from nonmotor symptoms like depression, fatigue, sleep disturbances, or pain, but there are no conclusive results of its effectiveness. ${ }^{16}$ However, to our knowledge, there is no such unit as the one described in this paper.

We believe that patients with end-stage Parkinsonism and their caregivers are confronted with similar problems and needs as those with typical palliative diagnoses, like cancer. ${ }^{17}$ However, patients often do not want to identify themselves as having a terminal illness with a limited lifespan. ${ }^{18}$ In general, disease survival in PD patients differs between studies. One study showed a two-fold increased risk of death, with a mean age at death of 82 years, ${ }^{19,20}$ and another study demonstrated survival of PD patients to be similar to that in a control population, up to a disease duration of ten years. ${ }^{21}$ After ten years, these rates were followed by a rise in mortality, which could explain our figures. It has been shown that, in PD patients reaching Hoehn and Yahr Stage III, as in our patients, the patient's survival time is limited. ${ }^{22}$

The multidisciplinary palliation approach performed by our staff is probably beneficial to patients and their caregivers, as indicated by the low discharge rate of patients from the unit. It has also earlier been described that non-neurologically educated health care personnel are unfamiliar with PD. ${ }^{23}$

The well-trained, specialized staff may also temper the impact of disease because there have been no acute transfers to hospital due to Parkinsonism. A recent study has reported that one-third of PD patients are dissatisfied with the way their PD was managed during an acute hospital stay. ${ }^{24}$ In contrast, that study also showed that PD patients are hospitalized in frequencies ranging from $7 \%$ to $29 \%$ per year, and 
that a substantial number of admissions may be prevented. In our PD permanent care unit it seems that the number of places is adequate with regard to demand, as indicated by the small waiting list.

The PD permanent care unit's work contrasts with that performed by families, which is categorized as a simple caring role with adjunctive professional services regularly or on demand. The content of the PD permanent care unit is more of complex care, continuously under surveillance by trained health care professionals, and also often involving advanced medical tasks in accordance with the concept of stroke units. ${ }^{25}$ Another study has emphasized that the nature of the disease will test the skills and coping abilities of everyone involved when caring for the PD patient. ${ }^{26}$ Clinical competence, commitment, and communication are three crucial parameters of the work process at the PD permanent care unit. This includes confirmation of diagnosis through medical records and ad hoc complementary investigations. The emphasis of the services is on quality of life. Individual and regular assessment of patients, with goal setting and follow-up, is crucial, as is the integration of family members into the caring process. A constant readjustment to a changing level of ability of the patient is important for the Parkinsonism patient. During the course of the disease, nutritional requirements often change, resulting in body weight gain or loss, which make a regular nutritional assessment important because it may affect the patient's quality of life. ${ }^{27}$ Moreover, common problem areas are well considered and handled, including careful observation for potentially contraindicated drugs, exact timing or even temporary cessation of drug administration, monitoring of complications due to immobilization, as well as monitoring of emerging psychiatric and cognitive dysfunction.

A limitation of our study is that we did not assess the impact of the PD permanent care unit on caregivers, ie, if they felt supported and/or relieved that their loved one's care was better. Moreover, we have no data on the health economic aspects of this model, ie, if it is cost-beneficial compared with traditional alternatives, like home care or care in a nonspecialized unit.

It is reasonable to believe that when patients are transferred from home to the PD permanent care unit, many aspects of caregiver burden are relieved. These include the physical, emotional, and social impact on caregivers, as well as the common limitations of personal time, eventually leading to decreased life space. ${ }^{28}$ It has been reported that caregiver burden increases with increasing disability and disease duration. ${ }^{29,30}$ The PD permanent care unit may therefore function as a relief of responsibilities for caregivers, although they are a valuable source of communication and are also very familiar with the patient and can help to identify the patient's needs. ${ }^{31}$ This is especially important for PD patients, because the symptoms of PD can vary widely between doses of medication and the side effects can be complex to manage.

Meeting all the needs of patients with Parkinsonism in a chronic/palliative state is an important aim of the PD permanent care unit. Despite the chronic/palliative state of these patients, there are many therapeutic options, with the overriding objective to let the patients end their days in a professional and comfortable environment. Future studies should include assessment of patients' and caregivers' quality of life and the impact on caregivers' life. A health-economic calculation, including the costs of caregivers, should also be integrated in such a study.

\section{Acknowledgment}

Many thanks to all staff members and especially the two nurses, Birgitta Wiklund and Lena Hultgren, of the PD permanent care unit who performed many of the assessments and provided much of the data.

\section{Disclosure}

The study was not supported by any monetary grants. The author has no connections with the pharmaceutical industry or stock companies which could be considered to be potential areas of conflict.

\section{References}

1. Maetzler W, Liepelt I, Berg D. Progression of Parkinson's disease in the clinical phase: Potential markers. Lancet Neurol. 2009;8:1158-1171.

2. Chaudhuri KR, Schapira AH. Non-motor symptoms of Parkinson's disease: Dopaminergic pathophysiology and treatment. Lancet Neurol. 2009;8:464-474.

3. Poewe W. Clinical measures of progression in Parkinson's disease. Mov Disord. 2009;24 Suppl 2:S671-S676.

4. Aarsland D, Marsch L, Schrag A. Neuropsychiatric symptoms in Parkinson's disease. Mov Disord. 2009;24:2175-2186.

5. Aarsland D, Larsen JP, Tandberg E, Laake K. Predictors of nursing home placement in Parkinson's disease: A population-based prospective study. J Am Geriatr Soc. 2000;48:938-942.

6. Lanoix M. Palliative care and Parkinson's disease: Managing the chronic-palliative interface. Chronic Illn. 2009;5:46-55.

7. Christakis N, Lamont E. Extent and determinants of error in doctors' prognoses in terminally ill patients: Prospective study cohort. $B M J$. 2000;320:469-473.

8. Clough CG, Blockley A. Palliative care for Parkinson's. In: Voltz R, Bernat JL, Borasio GD, Maddocks I, Oliver D, Portenoy RK, editors. Palliative Care in Neurology. New York: Oxford University Press; 2004.

9. Hoehn MM, Yahr MD. Parkinsonism: Onset, progression and mortality. Neurology. 1967;17:427-442.

10. Cockrell JR, Folstein MF. Mini-Mental State Examination (MMSE). Psychopharmacol Bull. 1988;24:689-692. 
11. Lees AJ, Hardy J, Revescz T. Parkinson's disease. Lancet. 2009;373: 2055-2066.

12. Keus SH, Bloem BR, Hendriks EJ, et al. Evidence-based analysis of physical therapy in Parkinson's disease with recommendations for practice and research. Mov Disord. 2007;22:451-460.

13. Downton JH, Andrews K. Prevalence, characteristics and factors associated with falls among the elderly living at home. Aging. 1991;3: 219-228.

14. Waters CH. Treatment of advanced stage patients with Parkinson's disease. Parkinsonism Relat Disord. 2002;9:15-21.

15. Rockwood K, Syolee P, McDowell I. Factors associated with institutionalization of older people in Canada: Testing a multifactorial definition of frailty. J Am Geriatric Soc. 1996;144:578-582.

16. Mallick S. Palliative care in Parkinson's disease: Role of cognitive behavior therapy. Indian J Palliat Care. 2009;15:51-56.

17. Hudson PL, Toye C, Kristjanson LJ. Would people with Parkinson's disease benefit from palliative care? Palliat Med. 2006;20:87-94.

18. Hudson PL, Elman LB, Houghton DJ, et al. Palliative care in amyotrophic lateral sclerosis, Parkinson's disease, and multiple sclerosis. J Palliat Med. 2007;10:433-457.

19. Morgante L, Salemi G, Meneghini F, et al. Parkinson disease survival: A population-based study. Arch Neurol. 2000;57:507-512.

20. Fall PA, Saleh A, Fredrickson M, Olsson, Granérus AK. Survival time, mortality, and cause of death in elderly patients with Parkinson's disease: A 9-year follow-up. Mov Disord. 2003;18:1312-1316.

21. Diem-Zangler A, Seppi K, Wenning G, et al. Mortality in Parkinson's disease: A 20-year follow-up study. Mov Disord. 2009;24:819-825.
22. Roos RA, Jongen JC, van der Velde EA. Clinical course of patients with idiopathic Parkinson's disease. Mov Disord. 1996;11:236-242.

23. Martignoni E, Godi L, Citterio A, et al. Comorbid disorders and hospitalization in Parkinson's disease: A prospective study. Neurol Sci. 2004;25:66-71.

24. Gerlach O, Winogrodzka A, Weber W. Clinical problems in the hospitalized Parkinson's disease patient: Systematic review. Mov Disord. January 31, 2011. [Epub ahead of print].

25. Bunting-Perry LK. Palliative care in Parkinson's disease: Implications for neuroscience nursing. J Neurosci Nurs. 2006;38:106-113.

26. Vergenz S. Caring for the Parkinson's patient: A nurse's perspective. Dis Mon. 2007;53:243-251.

27. Barichella M, Cereda E, Pezzoli G. Major nutritional issues in the management of Parkinson's disease. Mov Disord. 2009;24:1881-1892.

28. Lökk J. Reduced life-space of non-professional caregivers to Parkinson's disease patients with increased disease duration. Clin Neurol Neurosurg. 2009;111:583-587.

29. Schrag A, Morey D, Quinn N, Jahanshahi M. Impact of Parkinson's disease on patients'adolescent and adult children. Parkinsonism Relat Disord. 2004;10:391-397.

30. Lökk J. Caregiver strain in Parkinson's disease and the impact of disease duration. Eur J Phys Rehabil Med. 2008;44:39-45.

31. Goy ER, Carter JH, Ganzini L. Parkinson's disease at end of life: Caregiver perspectives. Neurology. 2007;69:611-612.
Journal of Multidisciplinary Healthcare

\section{Publish your work in this journal}

The Journal of Multidisciplinary Healthcare is an international, peerreviewed open-access journal that aims to represent and publish research in healthcare areas delivered by practitioners of different disciplines. This includes studies and reviews conducted by multidisciplinary teams as well as research which evaluates the results or conduct of such teams

\section{Dovepress}

or healthcare processes in general. The journal covers a wide range of areas and welcomes submission from practitioners at all levels, from all over the world. The manuscript management system is completely online and includes a very quick and fair peer-review system. Visit http://www.dovepress.com/ testimonials.php to read real quotes from published authors. 